\title{
Direct Visualization of Molecule Deprotonation on an Insulating Surface
}

\author{
Markus Kittelmann, ${ }^{\dagger}$ Philipp Rahe, ${ }^{\dagger, \ddagger}$ André Gourdon, ${ }^{\S}$ and Angelika Kühnle ${ }^{\dagger, *}$ \\ ${ }^{\dagger}$ Institut für Physikalische Chemie, Johannes Gutenberg-Universität Mainz, Duesbergweg 10-14, 55099 Mainz, Germany, and ${ }^{\S}$ Nanoscience Group, CNRS, \\ CEMES, 29 Rue J. Marvig, 31055 Toulouse, France. " Present address: Department of Physics and Astronomy, The University of Utah, 337 JFB, 115 South 1400 East, \\ Salt Lake City, UT 84112-0830.
}

F undamental understanding of deposition, diffusion, self-assembly, and reaction of molecules on surfaces is of utmost importance for a large number of daily life applications including catalysis ${ }^{1}$ and fabrication of functional surface coatings such as for organic solar cells as well as for smart responsive films. ${ }^{2,3}$ Consequently, adsorption, diffusion, and structure formation of organic molecules have been studied for decades, mainly focusing on metallic substrates. ${ }^{4,5}$ Very recently, on-surface reactions (see ref 6 and references therein) as well as chemical transformations including deprotonation ${ }^{7-9}$ have been addressed, revealing important insights into fundamental reaction steps.

For many applications, however, it becomes increasingly interesting to extend the knowledge gained on metallic surfaces to bulk insulator substrates, as many applications, e.g., catalysis and organic optoelectronics, require nonmetallic and electronically insulating support materials. Interestingly, fundamental processes such as molecular structure formation can be very different on bulk insulator compared to metal surfaces. ${ }^{10-12}$ For example, the typically weak molecule-surface interaction in the case of an insulating substrate favors molecular dewetting, ${ }^{13,14}$ which, in turn, can result in very complex molecular structures that are not observed on metallic surfaces. ${ }^{15-17}$ Nevertheless, comparatively little is known so far about molecule-surface interactions as well as chemical transformations and reactivity of organic molecules on electrically insulating surfaces. By making use of the Kelvin probe force microscopy (KPFM) technique, ${ }^{18}$ noncontact atomic force microscopy (NC-AFM) allows for directly mapping the charge state of adsorbates ${ }^{19}$ as well as the charge distribution within a single molecule. ${ }^{20}$ Only very recently, this technique has been applied for investigating organic molecules on bulk insulator surfaces, for example, for differentiating two

\section{ABSTRACT}
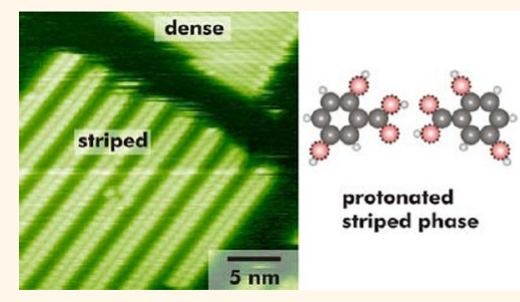

protonated striped phase

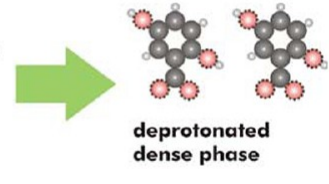

Elucidating molecular-scale details of basic reaction steps on surfaces is decisive for a fundamental understanding of molecular reactivity within many fields, including catalysis and on-surface synthesis. Here, the deprotonation of 2,5-dihydroxybenzoic acid (DHBA) deposited onto calcite $(101 ; \overline{4})$ held at room temperature is followed in situ by noncontact atomic force microscopy. After deposition, the molecules form two coexisting phases, a transient striped phase and a stable dense phase. A detailed analysis of high-resolution noncontact atomic force microscopy images indicates the transient striped phase being a bulk-like phase, which requires hydrogen bonds between the carboxylic acid moieties to be formed. With time, the striped phase transforms into the dense phase, which is explained by the deprotonation of the molecules. In the deprotonated state, the molecules can no longer form hydrogen bonds, but anchor to the surface calcium cations with their negatively charged carboxylate group. The deprotonation step is directly confirmed by Kelvin probe force microscopy images that unravel the change in the molecular charge.

KEYWORDS: deprotonation - insulating surface - noncontact atomic force microscopy $\cdot$ Kelvin probe force microscopy

adsorption geometries of triphenylene derivatives on $\mathrm{KBr}(001){ }^{21}$

Here we benefit from both the highresolution imaging capability of NC-AFM and the possibility to detect changes in the local charge distribution by KPFM for the direct visualization of the deprotonation step of 2,5-dihydroxybenzoic acid (DHBA) on a bulk insulating substrate, namely, calcite (see Figure 1). Upon deposition onto the natural $(101 ; \overline{4})$ cleavage plane held at room temperature, two molecular structures are observed to coexist. One of these structures closely resembles the molecular bulk structure, which is stabilized by
*Address correspondence to
kuehnle@uni-mainz.de.

Received for review June 12, 2012 and accepted July 27, 2012.

Published online July 27, 2012 10.1021/nn3025942

() 2012 American Chemical Society 
(a)

(b)
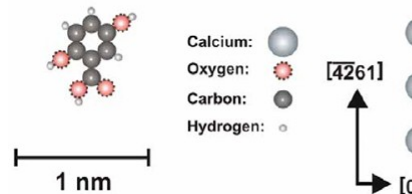

Figure 1. Model of (a) a 2,5-dihydroxybenzoic acid (DHBA) molecule and (b) the calcite $(101 ; 4)$ surface. The $\mathrm{CaCO}_{3}$ $(101 ; \overline{4})$ surface has a rectangular unit cell of size $5.0 \times 8.1$ $\AA$, consisting of two carbonate groups and two calcium ions. The carbonate groups are rotated such that one oxygen lies above, one in, and one below the plane spanned by the calcium ions.

intermolecular hydrogen bonds, thus, giving clear evidence for the fact that the molecules are still in the protonated state. Interestingly, this structure is observed to transform with time into the second structure, which is characterized by a dense packing of upright standing molecules. This configuration can be explained by deprotonated molecules anchoring toward the surface calcium cations. Thus, we are able to directly follow the deprotonation step of a carboxylic acid moiety on an insulating surface in situ, which is of great importance for elucidating molecularscale details of molecular reactivity on surfaces within fields such as on-surface synthesis ${ }^{6}$ and catalysis. ${ }^{22}$

\section{RESULTS AND DISCUSSION}

Upon submonolayer deposition of DHBA onto a calcite $(101 ; \overline{4})$ substrate held at room temperature, initially two distinctly different molecular phases coexist on the surface, as shown in Figure 2a. In this image, the majority of the surface is covered by a striped phase, which is characterized by islands formed from molecular double rows running along the $[4 ; \overline{2} ; \overline{6} 1]$ substrate direction. The outer shape of these islands exhibits a large number of kink sites. Moreover, several defects formed by missing molecules are evident within the striped phase. This striped layer constitutes a transient phase that transforms with time into a coexisting second phase. In the upper part of Figure 2a, an island of this second phase is seen, formed by densely packed molecules. The outline of this dense phase is less fragmented than the outline of the striped phase; however, kinks and vacancy islands are revealed for the dense phase as well. A closer view of both the striped and the dense phase is given in Figure $2 \mathrm{~b}$. In the lower part of this image, an island of the striped phase is shown, clearly revealing the molecular double-row structure and a single-molecule defect (circle). The upper part of Figure $2 b$ is covered by an island of the dense phase, unraveling a rectangular internal structure that is characterized by a dense packing of molecules.

To elucidate the molecular arrangement within both phases, we analyze high-resolution NC-AFM images of
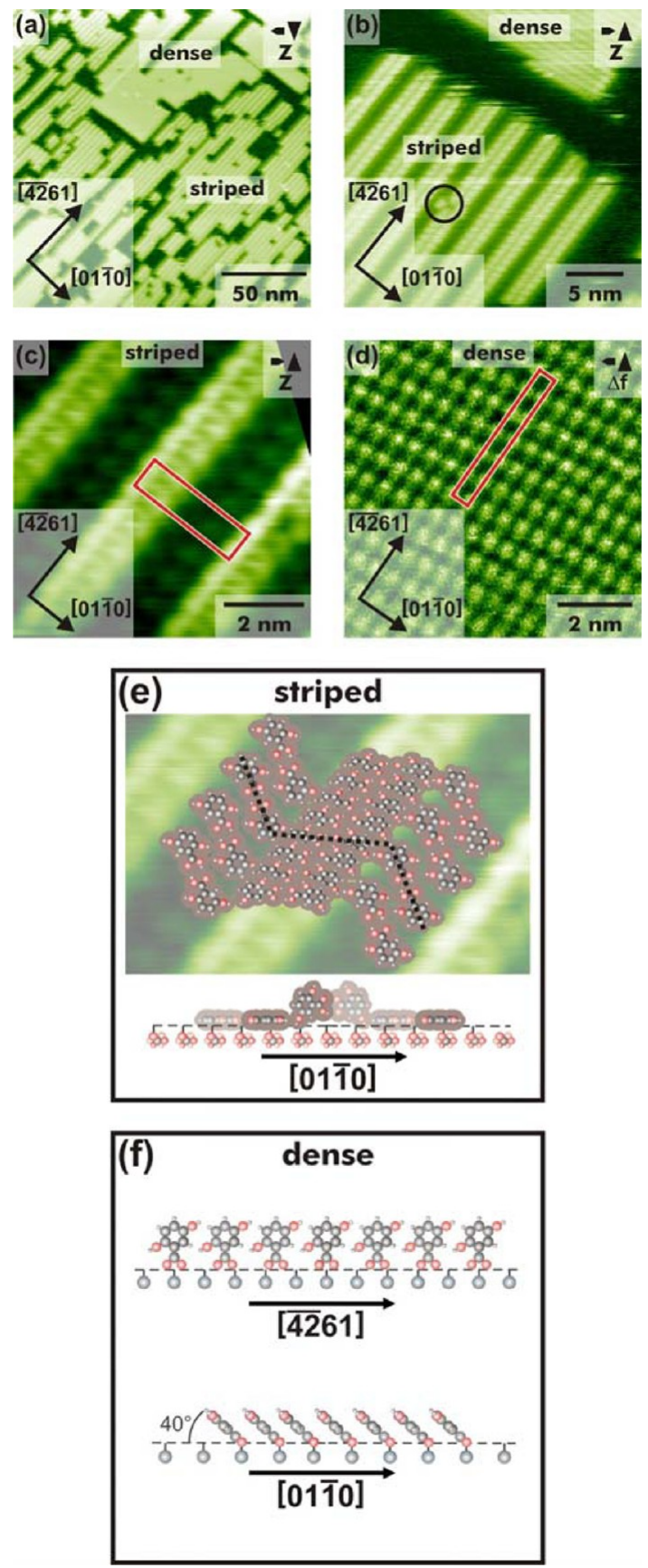

Figure 2. Coexisting molecular structures after deposition of DHBA onto calcite $(101 ; 4)$ held at room temperature. (a) Overview NC-AFM image revealing two coexisting phases, a defect-rich striped phase and a dense phase. (b) Closer view of both, the striped phase (lower part) and the dense phase (upper part). A defect in the striped phase is marked by a circle. (c) Drift-corrected high-resolution image of the striped phase, unraveling molecular double rows running along the $[4 ; 2 ; \overline{6} 1]$ direction and a molecular substructure in between the rows. The $(6 \times 1)$ unit cell is marked. (d) Driftcorrected high-resolution image of the dense phase, exhibiting a $(1 \times 5)$ superstructure. (e) Striped phase with superimposed model of the molecular bulk structure. The dashed line marks the molecules shown in the model at the bottom of the subfigure. (f) Model of the molecular arrangement within the dense phase. Note that we provide a simplified model with all aromatic rings aligned in one plane along the $[4 ; 2 ; 61]$ direction. 
the striped and dense phase shown in Figure $2 c, d$. In Figure $2 c$, the striped phase is presented, revealing an internal structure within the bright molecular rows as well as in between these rows. The different brightness indicates this structure being formed by molecules adopting different adsorption geometries. Our data suggest that the area in between the bright rows is covered by flat-lying molecules, while the bright molecular rows are formed by molecules with the molecular plane not parallel to the surface, resulting in an apparent height of approximately $0.4 \mathrm{~nm}$. This motif closely resembles the molecular arrangement of the bulk structure in the ordered form ${ }^{23}$ as superimposed in Figure 2e. In the ordered bulk structure, the molecules pair to dimers by hydrogen bond formation between the carboxylic groups. These dimers arrange in rows having alternating dimer orientations. The interdimer interaction is governed by hydrogen bond formation and by $\pi-\pi$ interaction of adjacent molecules. The different dimer orientation is reflected in our images by the alternate appearance of flat-laying molecules and molecules being oriented with their main axis parallel to the surface but having the molecular plane oriented upright, as illustrated in Figure $2 \mathrm{e}$. The striped phase constitutes a $(6 \times 1)$ superstructure, having unit cell dimensions of 2.99 and $0.81 \mathrm{~nm}$ along the $[011 ; \overline{0}]$ and $[4 ; \overline{2} ; \overline{6} 1]$ direction, respectively. Compared to the bulk structure, this superstructure corresponds to an extension of 13 and $8 \%$ along these two directions, indicating the subtle influence of the substrate on the resulting bulk-like structure. The fact that the striped phase constitutes a bulk-like structure provides experimental evidence for the conclusion that the molecules are still protonated within the striped phase, as the above-mentioned dimerization requires the carboxylic groups to be protonated.

Interestingly, with time the striped phase transforms into the dense phase, which remains as the only structure present after waiting for several hours to days. ${ }^{24} \mathrm{~A}$ high-resolution image of this phase is given in Figure 2d, exhibiting a densely packed structure having a rectangular unit cell with dimensions of $0.5 \mathrm{~nm} \times 4.1 \mathrm{~nm}$, corresponding to a $(1 \times 5)$ superstructure. The comparatively small lateral size of the individual features suggests this structure to be formed by molecules having their main axis oriented upright with respect to the surface normal as given in the model in Figure $2 \mathrm{f}$. In this configuration, intermolecular hydrogen bonding is no longer possible, providing a first indication for the deprotonation of the molecules. Moreover, the upright position can be readily explained by electrostatic interaction of deprotonated molecules with the surface calcium cations as detailed in the following. Along the $[011 ; \overline{0}]$ direction, the molecules follow the substrate periodicity, allowing for linking of the carboxylate with the substrate calcium cations. A further stabilization of this structure would be $\pi-\pi$ stacking of adjacent molecules along the $[011 ; \overline{0}]$ direction. To optimize the $\pi-\pi$ stacking distance, the molecules might tilt to bring the rings close to each other. To reach a favorable $\pi-\pi$ stacking distance of $0.34 \mathrm{~nm}$ in the parallel-displaced configuration, ${ }^{25}$ a tilt angle of approximately $40^{\circ}$ would be favorable, as obtained from simple trigonometry. This tilt would result in a reduction in molecular height from an upright height of approximately $0.77 \mathrm{~nm}$ to around $0.52 \mathrm{~nm}$, which is reflected by the experimentally observed height of $0.4 \mathrm{~nm}$. However, as height measurements with AFM rely on interaction forces, the height of chemically different species cannot be compared directly. ${ }^{26}$ Thus, the measured height can only provide an indication but no clear-cut experimental evidence for the proposed tilted geometry. ${ }^{27}$ Along the $[4 ; \overline{2} ; \overline{6} 1]$ direction, a compromise between moleculesurface interaction and intermolecular hydrogen bonding results in a molecular spacing that is responsible for a $(1 \times 5)$ superstructure. In the proposed geometry, six molecules are placed with their carboxylate groups atop 10 Ca cations, as shown in Figure $2 f$. This results in nonequivalent adsorption positions along the $[4 ; \overline{2} ; \overline{6} 1]$ direction. The NC-AFM results do not show different molecular appearance, which might be explained by two aspects. First, the NC-AFM might be sensitive only to the top part of the molecules, which is probably rather homogeneous. Second, the different adsorption positions might be compensated for by both molecule and surface relaxations. Regarding the intermolecular interaction along the $[4 ; \overline{2} ; \overline{6} 1]$ direction, a closest distance between the hydrogen atoms of approximately $0.15 \mathrm{~nm}$ is revealed. This geometry might be optimized by a slight rotation of the benzene ring around the $C-C$ bond between the carboxylate group and the benzene ring. These details are, however, beyond the scope of this simple model based on the NC-AFM images alone.

To confirm the above made tentative assignment of the dense phase being the deprotonated species, we made use of KPFM, allowing for a direct measurement of dipole moments and local charges. Deprotonated DHBA is negatively charged and, thus, should provide a clear signal in KPFM measurements. ${ }^{29}$ Protonated DHBA, on the other hand, carries a dipole (about $5 \mathrm{D}^{30}$ ) but is overall neutral. Consequently, for the protonated species we expect a less pronounced Kelvin signal as compared to the deprotonated molecules. In the absence of net charges, possible KPFM contrast can originate from surface charge transfer or intramolecular charge distribution. ${ }^{20}$ Resolving this charge distribution is rather difficult with KPFM because of the reduced lateral resolution of KPFM compared to NC-AFM. ${ }^{18}$ However, in some cases we succeeded in resolving an internal structure within the striped phase as discussed below. 

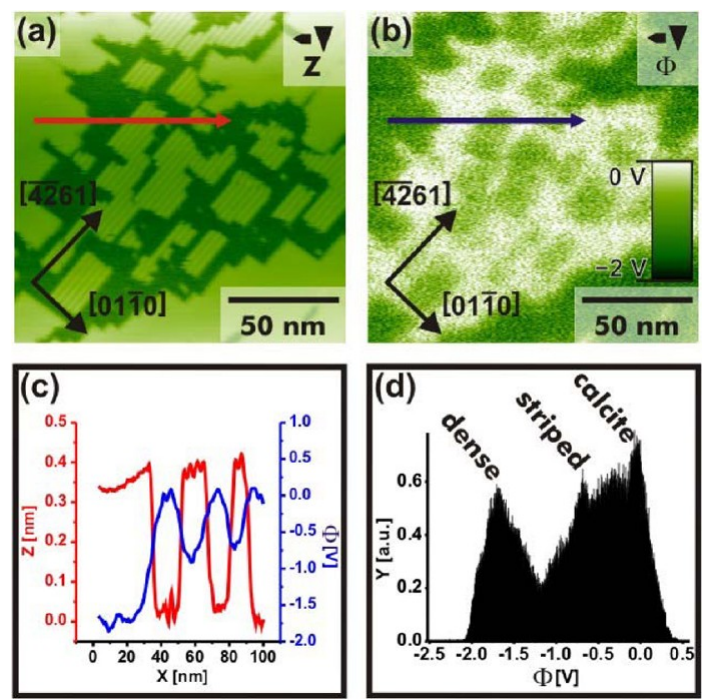

Figure 3. Kelvin probe force microscopy information. (a) NC-AFM topography image revealing the coexistence of the striped and the dense phase. (b) Corresponding KPFM image with the bare calcite surface set to $0 \mathrm{~V}$. Relative to the bare surface, the tip voltage above the stripe phase is shifted toward more negative values. Above the dense phase, the tip voltage is further shifted toward more negative values. (c) Height profile (red) and voltage profile (blue) from the indicated positions in (a) and (b). (d) Tip voltage histogram from (b), unraveling a peak at $0 \mathrm{~V}$ (substrate), $-0.75 \vee$ (striped phase), and $-1.75 \vee$ (dense phase). A further minor peak at around $-0.25 \mathrm{~V}$ is tentatively ascribed to diffusing protonated species. ${ }^{28}$.

An NC-AFM topography image and the corresponding KPFM image are shown in Figure 3a,b. The KPFM voltage of the bare calcite substrate is set to zero. The voltage required to compensate the local contact potential difference relative to the bare substrate is given in Figure $3 \mathrm{~b}$ with darker regions corresponding to negative voltages. Note that all data presented herein show the voltage applied to the tip. Compared to the bare surface, the Kelvin signal of the striped phase is shifted toward negative voltages. This is evident from the height and voltage profiles given in Figure $3 c$ and quantified in the voltage histogram shown in Figure $3 \mathrm{~d}$. According to this histogram, the voltage measured above the striped phase is approximately $-0.75 \mathrm{~V}$. This shift might be explained by a slight electron transfer from the surface to the DHBA molecules, which would result in a slight negative charge of the molecules. To compensate the resulting electrostatic forces, a more negative voltage has to be applied to the tip.

Moreover, due to the intramolecular charge distribution, an internal structure within the striped phase might be resolved when imaging with a very sharp conductive tip. Indeed, in rare cases, the KPFM signal revealed an internal structure within the striped phase as shown in Figure 4. As can be seen from the comparison of the topography (Figure 4a) and KPFM (Figure $4 b$ ) images and the corresponding line scans (Figure 4c), a protruding feature in the height profile is associated with a negative shift of the KPFM signal.
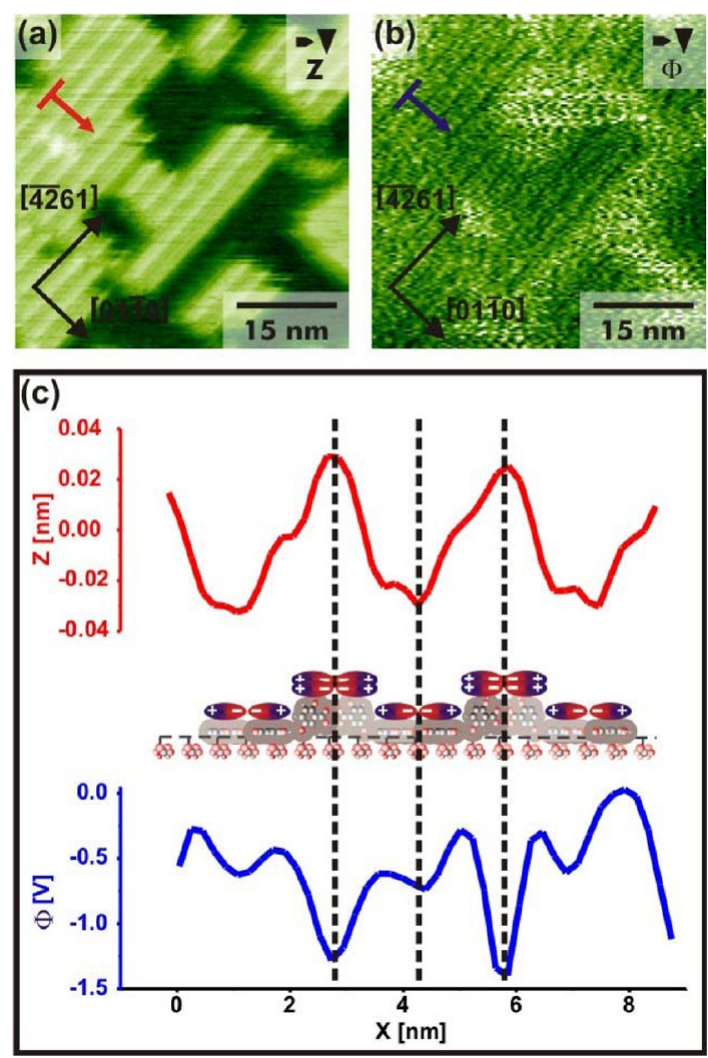

Figure 4. High-resolution KPFM image of the striped phase. (a) NC-AFM topography image showing a part of the striped phase. (b) Corresponding KPFM image. (c) Height profile (red) and KPFM profile (blue) taken along the indicated lines in (a) and (b). The profiles are averaged over 10 pixels, as illustrated by the perpendicular line at the beginning of the height profile lines in (a) and (b). The model illustrates the partial charge distribution due to molecular packing.

Additionally, a further small dip in the KPFM signal is seen in the minimum of the height profile. These characteristics can be explained by considering the molecular packing within the bulk-like phase, as illustrated in Figure 4c. Both, in the lying-down and standing-upright phase, the negative parts of the molecules are facing each other, leading to an accumulation of negative charges in the center of the structures. This is indeed reflected in the KPFM line profile in Figure 4c. The different magnitude of the negative shift can be understood by considering the different packing density within the standing-upright and lying-down parts. As the molecular packing is twice as large in the standing-upright part compared to the lying-down part, we expect a more pronounced signal in the protruding part in excellent agreement with the experimental observation.

Relative to the striped phase, the Kelvin signal of the dense phase is further shifted toward more negative values. As can be seen from the voltage histogram in Figure $3 d$, the voltage applied to the tip amounts to approximately $-1.75 \mathrm{~V}$ above the dense phase. This further shift can be readily assigned to the change in charge upon molecule deprotonation. After deprotonation, the molecules are negatively charged. To 
compensate for the electrostatic force acting between the molecules and the tip originating from this negative charge, a negative voltage has to be applied to the tip. This is directly expressed in the further shift toward more negative voltages above the dense phase compared to the striped phase. As the deprotonation of DHBA on calcite constitutes an acid-base reaction, we expect the proton to be linked to a surface carbonate group, forming hydrogencarbonate. Thus, in the deprotonated case, a dipole moment pointing toward the surface is formed by the negatively charged molecule and the surface. Because entire instead of partial charges are present in the deprotonated case, a more pronounce signal is evident for the deprotonated compared to the protonated islands.

Thus, the KPFM results directly confirm the above made assignment of the striped and dense phase being formed from the protonated and deprotonated species, respectively.

\section{CONCLUSION}

In conclusion, we present direct evidence for molecule deprotonation on an insulating surface by NCAFM images and KPFM measurements. Our high-resolution NC-AFM images elucidate the transient striped phase of DHBA on calcite $(101 ; \overline{4})$ being a bulk-like $(6 \times 1)$ phase, which transforms with time into a dense $(1 \times 5)$ superstructure that is formed by uprightstanding molecules. This latter phase can be readily explained by a deprotonation step, as negatively charged molecules anchor with the carboxylate group toward a surface calcium cation. The deprotonation step is directly visualized using KPFM, providing clear-cut confirmation of the above-made assignment.

\section{METHODS}

Calcite Preparation. Optical quality calcite samples from Korth Kristalle $\mathrm{GmbH}$ (Kiel, Germany) are cleaved in situ, resulting in flat $(101 ; 4)$ cleavage planes. ${ }^{31}$ Right after cleavage, the crystals are heated to $480 \mathrm{~K}$ for about $1 \mathrm{~h}$ to remove surface charges.

Molecule Deposition. The molecules ( $>99.0 \%$ purity) are purchased from Aldrich (Munich, Germany) and thoroughly outgassed at $315 \mathrm{~K}$ for $50 \mathrm{~h}$ prior to use. The molecules are sublimated in situ onto the freshly prepared calcite surface held at room temperature using a home-built Knudsen cell. The images shown here are achieved after sublimation for $10 \mathrm{~min}$ with a cell temperature of $339 \mathrm{~K}$, corresponding to a flux of approximately 0.08 monolayers per minute.

NC-AFM Imaging. All experiments are carried out at room temperature under UHV conditions (base pressure $\leq 1 \times 10^{-10} \mathrm{mbar}$ ) using a VT AFM 25 atomic force microscope (Omicron, Taunusstein, Germany) operated in the frequency modulation noncontact mode (NC-AFM). The system is equipped with an easyPLL Plus controller and phase-locked loop detector (Nanosurf, Liestal, Switzerland) for oscillation excitation and signal demodulation. FM-KPFM measurements are performed by applying an AC voltage to the tip (frequency $1 \mathrm{kHz}$, amplitude $2.3 \mathrm{~V}$ ) and compensating the resulting electrostatic force with an offset DC voltage applied to the tip (Kelvin signal) using a digital lock-in amplifier with built-in feedback loop (HF2 from Zurich Instruments, Zurich, Switzerland). We use $n$-doped silicon cantilevers (NanoWorld, Neuchâtel, Switzerland) with resonance frequencies of around $300 \mathrm{kHz}$ (type PPP-NCH) excited to oscillation amplitudes of about $10 \mathrm{~nm}$. Prior to their use, the cantilevers were $\mathrm{Ar}+$ sputtered at 2 $\mathrm{keV}$ for $5 \mathrm{~min}$ to remove contaminants. Drift-corrected images presented here are carefully processed following a routine published elsewhere. ${ }^{32}$ Image type as well as fast and slow scan directions are given in the upper right corner in each image.

Conflict of Interest: The authors declare no competing financial interest.

Acknowledgment. Financial support from the German Research Foundation through Grants SFB 625 TP B17 and KU1980/ 5-1 "In situ Chemistry of Molecular Assemblies on Dielectric Surfaces" is gratefully acknowledged. We thank Sadik Hafizovic (Zurich Instruments AG) for stimulating discussions.

\section{REFERENCES AND NOTES}

1. Nilius, N.; Risse, T.; Schauermann, S.; Shaikhutdinov, S.; Sterrer, M.; Freund, H. J. Model Studies in Catalysis. Top. Catal. 2011, 54, 4-12.
2. Forrest, S. R. Ultrathin Organic Films Grown by Organic Molecular Beam Deposition and Related Techniques. Chem. Rev. 1997, 97, 1793-1896.

3. Umbach, E.; Glockler, K.; Sokolowski, M. Surface "Architecture" With Large Organic Molecules: Interface Order and Epitaxy. Surf. Sci. 1998, 402-404, 20-31.

4. Rosei, F.; Schunack, M.; Naitoh, Y.; Jiang, P.; Gourdon, A.; Lægsgaard, E.; Stensgaard, I.; Joachim, C.; Besenbacher, F. Properties of Large Organic Molecules on Metal Surfaces. Prog. Surf. Sci. 2003, 71, 95-146.

5. Barth, J. V. Transport of Adsorbates at Metal Surfaces: From Thermal Migration to Hot Precursors. Surf. Sci. Rep. 2000, 40, 75-149.

6. Franc, G.; Gourdon, A. Covalent Networks through onSurface Chemistry in Ultra-High Vacuum: State-of-the-Art and Recent Developments. Phys. Chem. Chem. Phys. 2011, 13, 14283-14292.

7. Papageorgiou, A. C.; Fischer, S.; Reichert, J.; Diller, K.; Blobner, F.; Klappenberger, F.; Allegretti, F.; Seitsonen, A. P.; Barth, J. V. Chemical Transformations Drive Complex Self-Assembly of Uracil on Close-Packed Coinage Metal Surfaces. ACS Nano 2012, 6, 2477-2486.

8. Kanninen, L.; Jokinen, N.; Ali-Loytty, H.; Jussila, P.; Lahtonen, K.; Hirsimaki, M.; Valden, M.; Kuzmin, M.; Parna, R.; Nommiste, E. Adsorption Structure and Bonding of Trimesic Acid on Cu(100). Surf. Sci. 2011, 605, 1968-1978.

9. Dai, P. X.; Chen, T.; Wang, D.; Wan, L. J. Potential Dependent Adsorption Geometry of 2,5-Dihydroxybenzoic Acid on a $\mathrm{Au}(111)$ Surface: An In Situ Electrochemical Scanning Tunneling Microscopy Study. J. Phys. Chem. C 2012, 116, 6208-6214.

10. Such, B.; Trevethan, T.; Glatzel, T.; Kawai, S.; Zimmerli, L.; Meyer, E.; Shluger, A. L.; Amijs, C. H. M.; de Mendoza, P.; Echavarren, A. M. Functionalized Truxenes: Adsorption and Diffusion of Single Molecules on the $\mathrm{KBr}(001)$ Surface. ACS Nano 2010, 4, 3429-3439.

11. Pawlak, R.; Nony, L.; Bocquet, F.; Olson, V.; Sassi, M.; Debierre, J. M.; Loppacher, C.; Porte, L. Supramolecular Assemblies of 1,4-Benzene Diboronic Acid on $\mathrm{KCl}(001)$. J. Phys. Chem. C 2010, 114, 9290-9295.

12. Rahe, P.; Nimmrich, M.; Greuling, A.; Schütte, J.; Stará, I. G.; Rybáček, J.; Huerta-Angeles, G.; Starý, l.; Rohlfing, M.; Kühnle, A. Toward Molecular Nanowires Self-Assembled on an Insulating Substrate: Heptahelicene-2-Carboxylic Acid on Calcite (10-14). J. Phys. Chem. C 2010, 114, 1547-1552.

13. Burke, S. A.; Topple, J. M.; Grütter, P. Molecular Dewetting on Insulators. J. Phys.: Condens. Matter 2009, 21, 423101.

14. Rahe, P.; Lindner, R.; Kittelmann, M.; Nimmrich, M.; Kühnle, A. From Dewetting to Wetting Molecular Layers: $C_{60}$ on 
$\mathrm{CaCO}_{3}(10-14)$ as a Case Study. Phys. Chem. Chem. Phys. 2012, 14, 6544-6548.

15. Burke, S. A.; Mativetsky, J. M.; Hoffmann, R.; Grütter, P. Nucleation and Submonolayer Growth of $\mathrm{C}_{60}$ on KBr. Phys. Rev. Lett. 2005, 94, 096102.

16. Burke, S. A.; Ji, W.; Mativetsky, J. M.; Topple, J. M.; Fostner, S.; Gao, H. J.; Guo, H.; Grütter, P. Strain Induced Dewetting of a Molecular System: Bimodal Growth of PTCDA on $\mathrm{NaCl}$. Phys. Rev. Lett. 2008, 100, 186104.

17. Körner, M.; Loske, F.; Einax, M.; Kühnle, A.; Reichling, M.; Maass, P. Second-Layer Induced Island Morphologies in Thin-Film Growth of Fullerenes. Phys. Rev. Lett. 2011, 107, 016101.

18. Zerweck, U.; Loppacher, C.; Otto, T.; Grafström, S.; Eng, L. M. Accuracy and Resolution Limits of Kelvin Probe Force Microscopy. Phys. Rev. B 2005, 71, 125424.

19. Gross, L.; Mohn, F.; Liljeroth, P.; Repp, J.; Giessibl, F. J.; Meyer, G. Measuring the Charge State of an Adatom with Noncontact Atomic Force Microscopy. Science 2009, 324, 1428-1431.

20. Mohn, F.; Gross, L.; Moll, N.; Meyer, G. Imaging the Charge Distribution within a Single Molecule. Nat. Nanotechnol. 2012, 7, 227.

21. Hinaut, A.; Pujol, A.; Chaumeton, F.; Martrou, D.; Gourdon, A.; Gauthier, S. An NC-AFM and KPFM Study of the Adsorption of a Triphenylene Derivative on $\operatorname{KBr}(001)$. Beilstein J. Nanotechnol. 2012, 3, 221-229.

22. Leydier, F.; Chizallet, C.; Chaumonnot, A.; Digne, M.; Soyer, E.; Quoineaud, A. A.; Costa, D.; Raybaud, P. Bronsted Acidity of Amorphous Silica-Alumina: The Molecular Rules of Proton Transfer. J. Catal. 2011, 284, 215-229.

23. Haisa, M.; Kashino, S.; Hanada, S. I.; Tanaka, K.; Okazaki, S.; Shibagaki, M. The Structures of 2-Hydroxy-5-Methylbenzoic Acid and Dimorphs of 2,5-Dihydroxybenzoic Acid. Acta Crystallogr., B 1982, 38, 1480-1485.

24. Alternatively, the sample was annealed to $440 \mathrm{~K}$, resulting in the same situation of the dense phase remaining exclusively on the surface.

25. Sinnokrot, M. O.; Valeev, E. F.; Sherrill, C. D. Estimates of the Ab Initio Limit for $\pi-\pi$ Interactions: The Benzene Dimer. J. Am. Chem. Soc. 2002, 124, 10887-10893.

26. Sadewasser, S.; Lux-Steiner, M. C. Correct Height Measurement in Noncontact Atomic Force Microscopy. Phys. Rev. Lett. 2003, 91, 266101.

27. Interestingly, as similar heights are revealed with the Kelvin controller enabled (see below), electrostatic interactions are demonstrated not to degrade the height measurements significantly.

28. This assignment is confirmed by comparison with KPFM images taken several hours after molecule deposition when no protonated species are left on the surface. Consequently, no diffusing protonated molecules are present in this case and the peak at $-0.25 \mathrm{~V}$ vanishes.

29. Vitali, L.; Levita, G.; Ohmann, R.; Comisso, A.; De Vita, A.; Kern, K. Portrait of the Potential Barrier at Metal-Organic Nanocontacts. Nat. Mater. 2010, 9, 320-323.

30. Huq, F. Molecular Modelling Analysis of the Metabolism of Aspirin. Asian J. Chem. 2007, 19, 102-108.

31. Tröger, L.; Schütte, J.; Ostendorf, F.; Kühnle, A.; Reichling, M. Concept for Support and Cleavage of Brittle Crystals. Rev. Sci. Instrum. 2009, 80, 063703.

32. Rahe, P.; Bechstein, R.; Kühnle, A. Vertical and Lateral Drift Corrections of Scanning Probe Microscopy Images. J. Vac. Sci. Technol., B 2010, 28, C4E31-C4E38. 